\title{
Xpert MTB/RIF assay for diagnosis of extrapulmonary tuberculosis in children: a systematic review and meta-analysis
}

\author{
Young Seok Seo, Ji-Man Kang, Dong Soo Kim and Jong Gyun Ahn ${ }^{*}$ [D
}

\begin{abstract}
Background: The Xpert ${ }^{\oplus}$ MTB/RIF assay (Xpert; Cepheid, Sunnyvale, CA, USA) is a cartridge-based nucleic acid amplification assay for rapidly diagnosing tuberculosis and assessing antibiotic sensitivity. Although previous evidence supports the use of Xpert for diagnosing extrapulmonary tuberculosis (EPTB) in adults, information regarding the accuracy of Xpert for EPTB only in children is lacking. This meta-analysis was performed to assess the accuracy of Xpert for detecting EPTB in children.

Methods: We searched the MEDLINE, EMBASE, and Cochrane Infectious Diseases Group Specialized Register from January 1, 2010 to July 16, 2019 for studies of the diagnostic performance wherein Xpert was analyzed against cultures or composite reference standards for $<18$-year-old children with EPTB.

Results: In only pediatric studies, 8 studies including 652 samples were selected. The pooled sensitivity and specificity of Xpert for all samples were $71 \%$ (95\% Cl 0.63-0.79) and 97\% (95\% Cl 0.95-0.99), respectively. The area under the summary receiver operating characteristic (sROC) curve was 0.89 . For lymph node tissues or aspirates, the pooled sensitivity and specificity of Xpert were $80 \%(95 \% \mathrm{Cl} 0.70-0.88)$ and $94 \%$ (95\% Cl 0.89-0.97), respectively; for cerebrospinal fluid (CSF), these values were $42 \%$ (95\% Cl 0.22-0.63) and 99\% (95\% Cl 0.95-1.00), respectively.

Conclusion: Overall, Xpert displayed high specificity but modest sensitivity across various samples for diagnosing pediatric EPTB compared to the composite reference standard. Xpert sensitivity varied with the sampling site and was especially lower in CSF samples. Positive Xpert results may be considered to indicate a presumptive case of pediatric EPTB, whereas negative test results indicate that the possibility of pediatric EPTB should not be excluded.
\end{abstract}

Keywords: Extrapulmonary tuberculosis, Xpert MTB/RIF, Child, Meta-analysis

\section{Background}

Tuberculosis (TB) is a serious threat to global public health and the leading cause of death from a single infectious disease worldwide, surpassing the rates of malaria and HIV/AIDS. Globally, there were an estimated 10.0 million incident cases of TB in 2018, with approximately 1 million (11\%) among children $<15$ years. However, the mortality rate was higher in children aged $<15$ years, accounting for $14 \%$ of total deaths, which is greater than that in incident cases, suggesting poorer access to diagnosis and treatment [1].

\footnotetext{
* Correspondence: JGAHN@yuhs.ac

Department of Pediatrics, Severance Children's Hospital, Yonsei University College of Medicine, 50-1 Yonsei-ro, Seodaemun-gu, Seoul 03722, South Korea
}

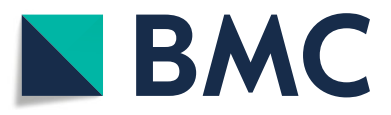

(c) The Author(s). 2020 Open Access This article is distributed under the terms of the Creative Commons Attribution 4.0 International License (http://creativecommons.org/licenses/by/4.0/), which permits unrestricted use, distribution, and reproduction in any medium, provided you give appropriate credit to the original author(s) and the source, provide a link to the Creative Commons license, and indicate if changes were made. The Creative Commons Public Domain Dedication waiver (http://creativecommons.org/publicdomain/zero/1.0/) applies to the data made available in this article, unless otherwise stated. curring within a location in the body other than the lungs (e.g., meninges, lymph nodes, pleura, abdomen, genitourinary tract, skin, joints, and bones) [2]. EPTB is estimated to account for $8-24 \%$ (15\% of the 7.0 million incident cases on average) of all TB infections worldwide [1]. These numbers vary in accordance with specific risk factors in certain regions, such as age, sex, concurrent HIV infection, and underlying comorbidities $[1,3]$. EPTB commonly occurs in children and HIV-infected individuals [4].

To diagnose EPTB, samples should be obtained from sites of suspected infection and cultured. The diagnosis of pediatric EPTB remains challenging because clinical specimens are potentially inaccessible for appropriate 
sampling and require invasive diagnostic procedures [5]. Furthermore, 8-12 weeks are required to obtain the results through culturing, thus delaying treatment [6]. Because of these diagnostic challenges among children, the incidence of pediatric EPTB is likely underestimated [7].

The Xpert ${ }^{\circ}$ MTB/RIF assay (Xpert; Cepheid, Sunnyvale, CA, USA) is a cartridge-based nucleic acid amplification assay for rapid TB diagnosis and rapid antibiotic sensitivity analysis. Currently, Xpert is used as a rapid assay for TB diagnosis as recommended by the World Health Organization (WHO) [1]. Since 2013, Xpert has also been recommended for diagnosing TB meningitis and TB lymphadenitis in children [8]. Several systematic reviews have been conducted to determine the diagnostic accuracy of Xpert for EPTB in both pediatric and adult populations; however, no studies have specifically evaluated for children [5, 9-16]. Therefore, data regarding the accuracy of Xpert exclusively among children are unavailable. We conducted a systematic review and meta-analysis to assess the diagnostic accuracy of Xpert for detecting EPTB among children.

\section{Methods}

Data sources and search strategies

We searched the MEDLINE, EMBASE, and Cochrane Infectious Diseases Group Specialized Register. Our last search was carried out on July 16, 2019. Furthermore, we manually reviewed the bibliographies of the included articles. The primary search terms were "Xpert," "GeneXpert," "Cepheid," "MTB/RIF," and "Tuberculosis." The search methodology applied for each database is shown in Additional file 1. The bibliography was screened for full-length research articles in all languages. Moreover, we reviewed the full-text to select articles describing the exclusive analysis of pediatric EPTB.

\section{Eligibility criteria}

The following inclusion criteria were used: (1) studies using Xpert as a diagnostic tool for detecting EPTB compared to a reference standard in each study, with all non-respiratory samples (i.e., lymph node aspirate or tissue, CSF, pleural fluid, etc.); (2) studies evaluating the diagnostic performance of Xpert; and (3) studies providing pediatric ( $0-18$ years) data. Studies were included regardless of HIV infection status.

We excluded reviews, letters, editorials, expert opinions, animal experiments, and studies that only presented an abstract. Studies that did not include separate pediatric data were also excluded. We attempted to include all types of EPTB samples; however, studies reporting the use of gastric lavage samples were excluded because they were intended to diagnose pulmonary TB. Studies including samples from fewer than five patients and studies with no or insufficient data to construct a $2 \times 2$ contingency table to determine sensitivity and specificity were also excluded. If data were obtained in more than one article from the same author, the article with the most data was selected.

\section{Study selection}

Two review authors (YS Seo and JK Ahn) independently assessed the titles and abstracts in accordance with the inclusion and exclusion criteria, followed by a full-text review of the selected studies. Discrepancies regarding the inclusion of articles between the two authors were resolved by the third author (DS Kim).

\section{Composite reference standard (CRS)}

To compare the accuracy of Xpert, mycobacterial culturing or a CRS was used as a reference standard. The CRS was defined by the authors of each study. Because of the paucibacillary characteristics of extrapulmonary TB, the clinical diagnosis of TB was also included. The CRS included histopathological, smearing, and clinical response analysis to treatment with anti-TB therapy along with culturing.

\section{Quality assessment}

Qualitative assessment was performed using the Quality Assessment of Diagnostic Accuracy Studies-2 (QUADAS-2) tool [17]. All eligible studies were evaluated based on four domains: patient selection, index test, reference standard, and flow and timing. Each domain was assessed in terms of the risk of bias, and the first three domains were assessed in terms of concerns regarding applicability.

\section{Statistical analysis}

We determined the sensitivity and specificity of Xpert with $95 \%$ confidence intervals compared to culturing or the CRS. To assess the heterogeneity among studies, the chi-square test was performed. Heterogeneity was defined as a $p$-value of $<0.10$. In case of heterogeneity, different thresholds were considered to influence sensitivity and specificity. To assess the presence of a threshold effect, Spearman's $\rho$ correlation analysis was performed with $\rho>0.6$ indicating a threshold effect. The sensitivities and specificities of Xpert in each study were determined and subjected to meta-analysis with a bivariate random-effects model. We plotted the summary receiver operating characteristic (sROC) curve with this model. R-package mada (version 3.5.1.) was used to generate forest plots and an ROC curve.

\section{Results}

\section{Identified studies}

Figure 1 shows the protocol for the screening of articles. Of the 2225 articles obtained from MEDLINE $(n=844)$, 


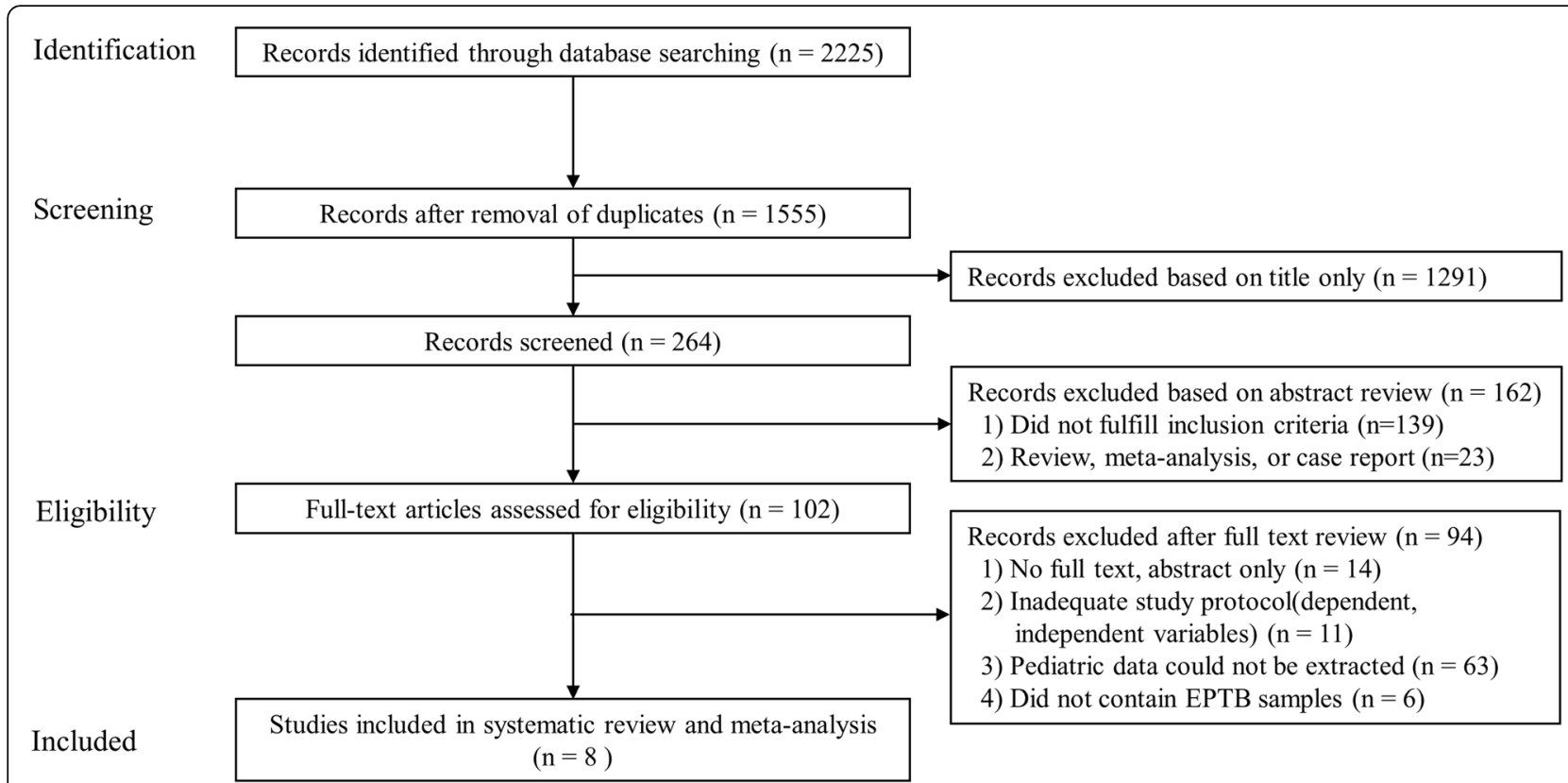

Fig. 1 Flow diagram showing the protocol for study selection. EPTB, extrapulmonary tuberculosis

EMBASE $(n=1180)$, and Cochrane $(n=201), 670$ duplicates were excluded. After screening the titles, 1291 studies were excluded. After screening the abstracts, 162 studies were excluded for the following reasons: 139 studies did not fulfill the inclusion criteria and 23 studies were excluded because they were review studies, metaanalysis studies, or case reports. After reviewing the fulltext of the remaining 102 studies, 94 studies were excluded for the following reasons: 14 studies included only abstracts, 6 studies did not contain EPTB samples, 11 studies had inadequate study protocols, and 63 studies did not separate pediatric data. Finally, we identified 8 studies that included 652 samples.

\section{Study characteristics}

Table 1 summarizes the characteristics of the included studies. Five studies were conducted in Africa [18-22], 1 study in India [23], 1 study in South Korea [24], and 1 in Italy [25]. Two studies were retrospective [24, 25] and the remaining 6 were prospective [18-23]. All articles were written in English. Sample numbers varied in accordance with the studies. In total, 277 lymph nodes, 218 CSF samples, 20 pleural fluid samples, and 137 musculoskeletal samples were reviewed.

\section{Quality assessment}

Quality assessment was performed using QUADAS-2, as summarized in Fig. 2. In the patient selection domain, one study reported a high risk of bias, wherein patients were selected through convenience [18]. Other studies reported a low risk of bias. Regarding applicability, one study [18] had low concern because patients were assessed in a local hospital; another study [20] revealed high concern because only inpatients were evaluated at a tertiary-care center $[18,20]$. Other studies reported unclear concern because of a lack of enough information regarding the clinical setting $[19,21-25]$. For the index test and reference standard, the included studies generally had a low risk of bias and low applicability concerns.

\section{Meta-analysis for diagnostic accuracy of Xpert}

The 8 studies were evaluated as described above (Fig. 3). Regardless of the sample type, the pooled sensitivity and specificity of all samples were $71 \%$ (95\% CI $0.63-0.79$ ) and $97 \%$ (95\% CI 0.95-0.99), respectively. The area under the ROC curve was 0.89 (Fig. 4). High heterogeneity was confirmed through chi-square analysis for both sensitivity and specificity. However, it was difficult to assign statistical significance to the data because of the heterogeneity among sample types. Therefore, each sample was divided into subgroups.

\section{Detection of lymph node TB}

Six studies used Xpert to analyze lymph node samples obtained by fine-needle aspiration biopsy (FNAB) or lymph node biopsy rather than a CRS [18, 19, 22-25]. The pooled sensitivity and specificity of the lymph node samples were $80 \%$ (95\% CI 0.70-0.88) and 94\% (95\% CI $0.89-0.97)$, respectively. The area under the ROC curve was 0.92 . High heterogeneity was confirmed using the chi-square test for specificity. 


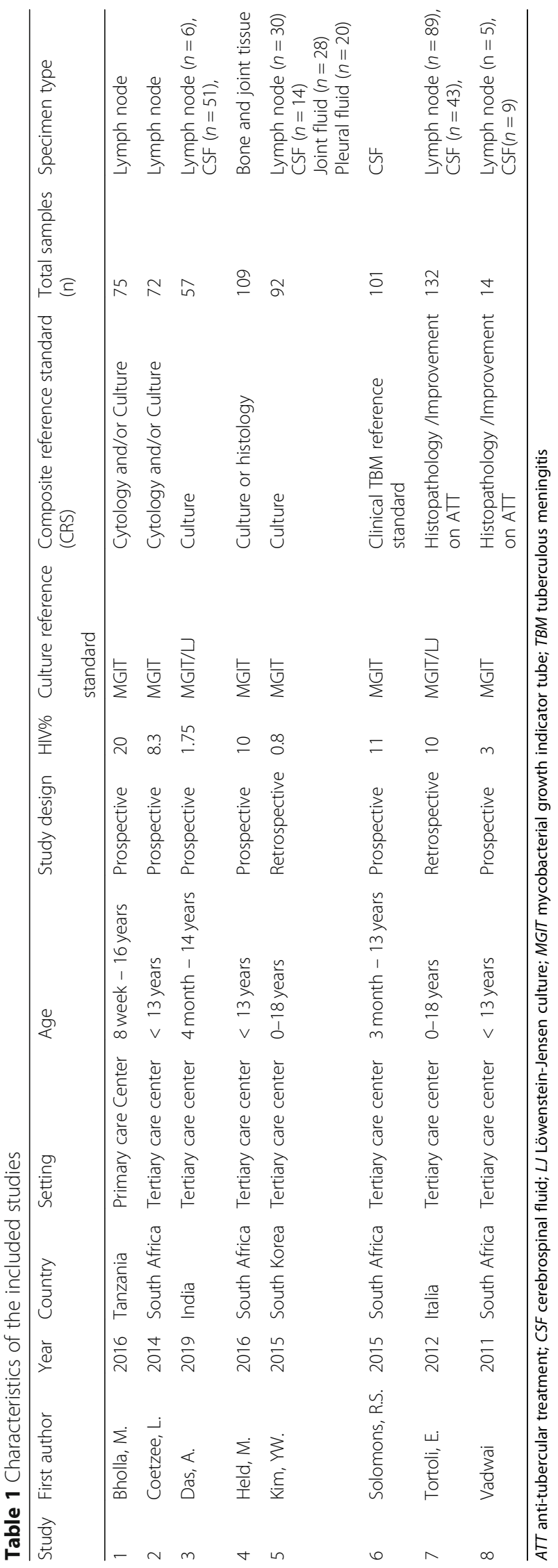




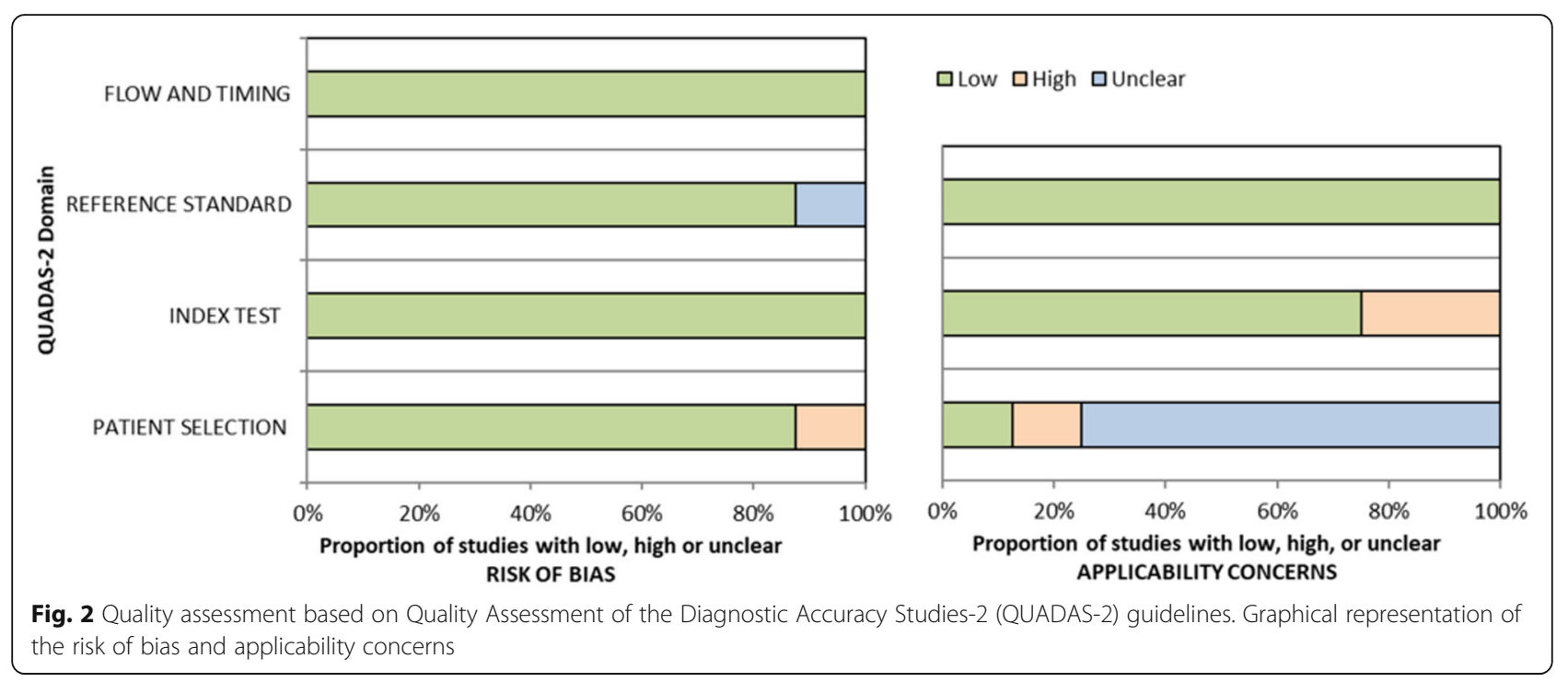

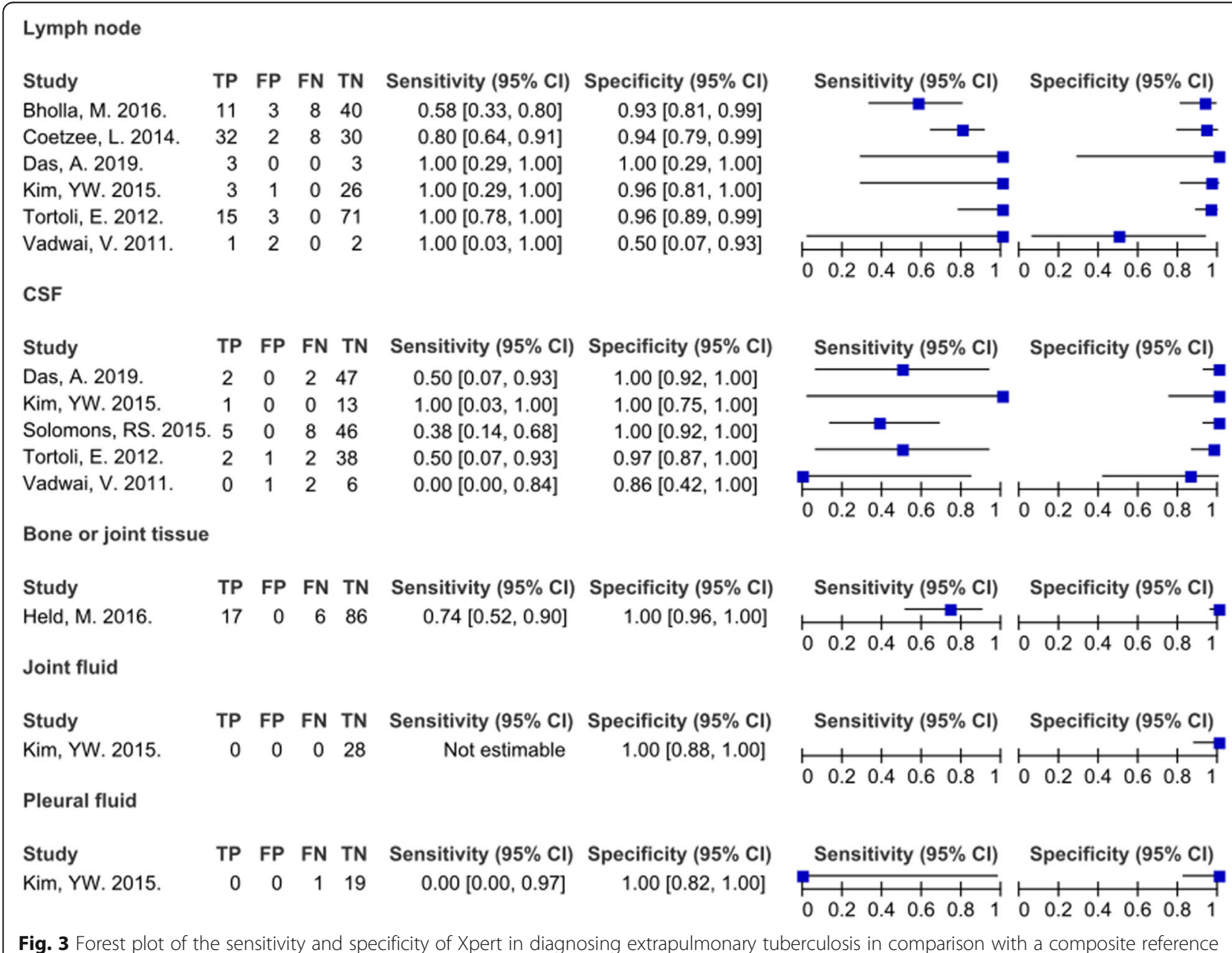
standard in accordance with the study and specimen type. TP, true-positive; FP, false-positive; FN, false-negative; TN, true-negative 


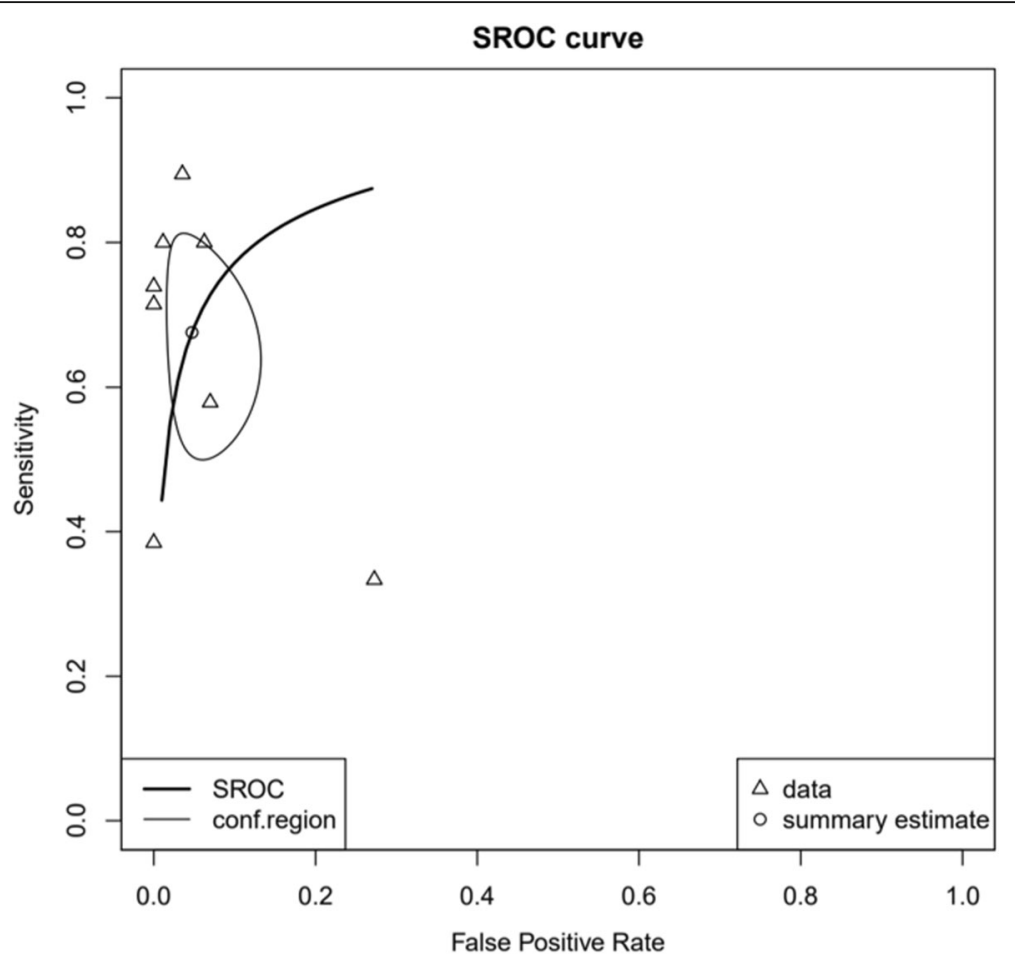

Fig. 4 Hierarchical summary receiver operating characteristic (HSROC) curve of the diagnostic accuracy of Xpert ${ }^{\oplus}$ MTB/RIF assay (Xpert) for extrapulmonary tuberculosis (EPTB). Summary points of the sensitivity and specificity, HSROC curve, and 95\% confidence intervals. The area under the curve of the HSROC for Xpert was 0.89

\section{Detection of TB meningitis}

Five studies used Xpert to analyze CSF samples rather than a CRS [21-25]. The pooled sensitivity and specificity of the CSF samples were $42 \%$ (95\% CI 0.22-0.63) and $99 \%$ (95\% CI $0.95-1.00)$, respectively. The area under the ROC curve was 0.57 . High heterogeneity was confirmed through chi-square analysis for specificity.

\section{Comparison with other published meta-analyses}

Nine meta-analyses have been performed to assess the accuracy of Xpert for detecting EPTB (Table 2) [5, 9-16]. These reviews evaluated data from both children and adult populations; however, no studies analyzed children alone. In these analyses, the diagnostic accuracy of Xpert in lymph node samples showed sensitivities ranging from 83 to $96 \%$ and specificities ranging from 86 to $94 \%(80.2$ and $94.0 \%$ in this study) $[5,10,12,13,16]$. In CSF samples, the sensitivities ranged from 69 to $85 \%$ and specificities ranged from 97 to $100 \%$ (41.7 and $98.7 \%$ in this study) $[5,10,12,13]$. In the pleural fluid samples, the sensitivities ranged from 34 to $51.4 \%$ and specificities ranged from 98 to $99 \%[5,10,12-14]$. In bone or joint tissue specimens, sensitivities and specificities ranged from 84 to $91.8 \%$ and 82 to $98 \%$, respectively $[10,15]$. In bone or joint pus specimens, the sensitivity was $82 \%$ and specificity was 99\% [15]. In the joint fluid specimens, sensitivity and specificity were $97.2 \%$ and from $90.2 \%$, respectively [10]. However, in this study, meta-analysis of pleural TB and bone or joint TB could not be performed because of the small number of studies.

\section{Discussion}

The present study summarizes the overall performance of Xpert for diagnosing pediatric EPTB based on the currently available literature. Although previous systematic reviews analyzed data from both children and adult populations, no studies have reported distinct data for children [5, 9-16]. This study shows that Xpert has high specificity in pediatric EPTB, although its sensitivity is relatively lower and highly variable among specimen types.

In a recent meta-analysis reporting data primarily about adults, the pooled sensitivity varied among different types of specimens (83.1\% in lymph node aspirates, $71.1 \%$ in CSF, and $94.6 \%$ in bone or joint tissue). However, the pooled specificity was relatively high between sample types (86\% in lymph node aspirates, $98 \%$ in CSF, and $85.3 \%$ in bone or joint tissue) [10]. These data agree with the present results in that Xpert showed high specificity among various specimens in our study. Furthermore, the pooled sensitivity varied among the different types of specimens in this study. Overall, however, the 


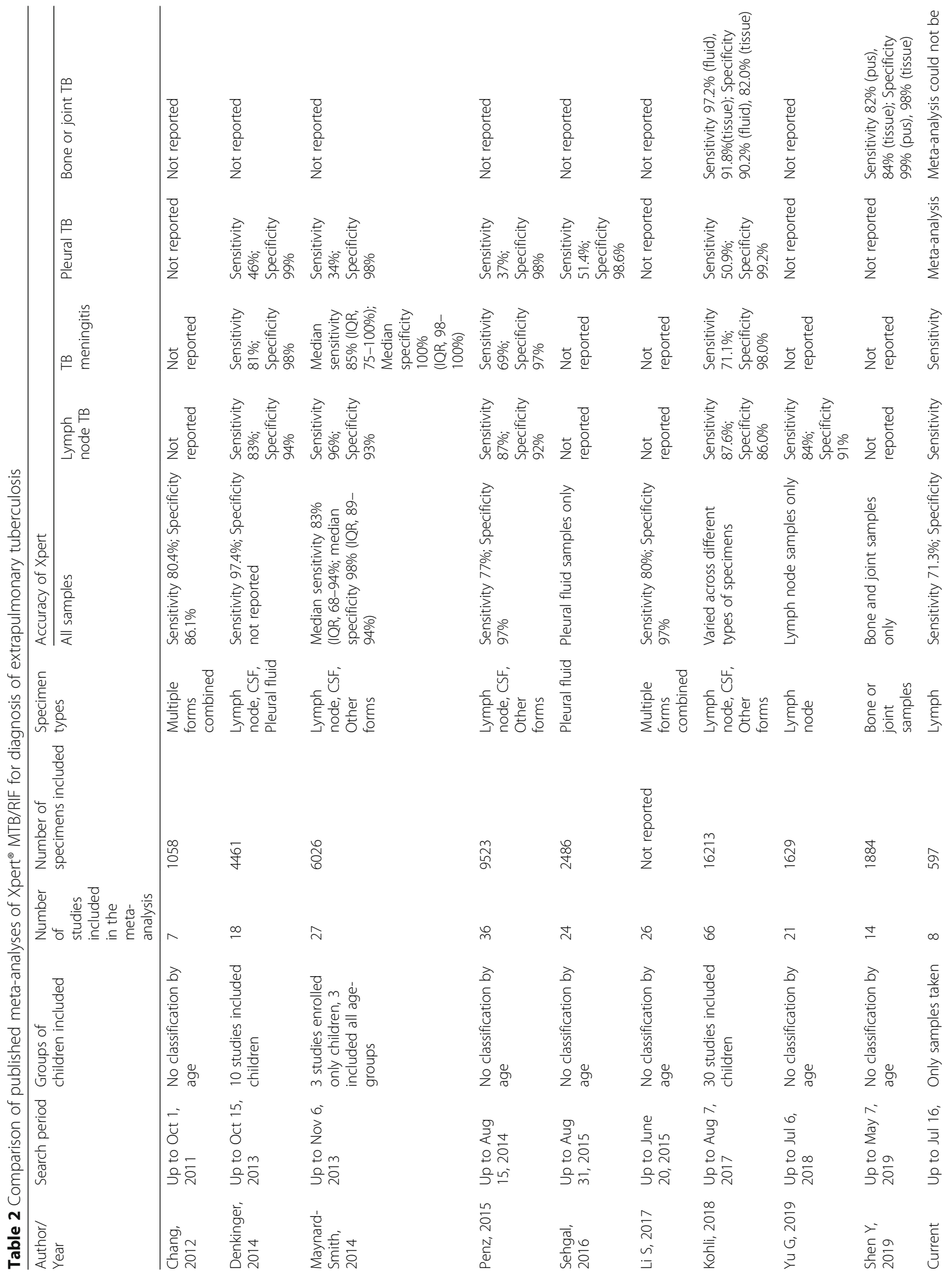




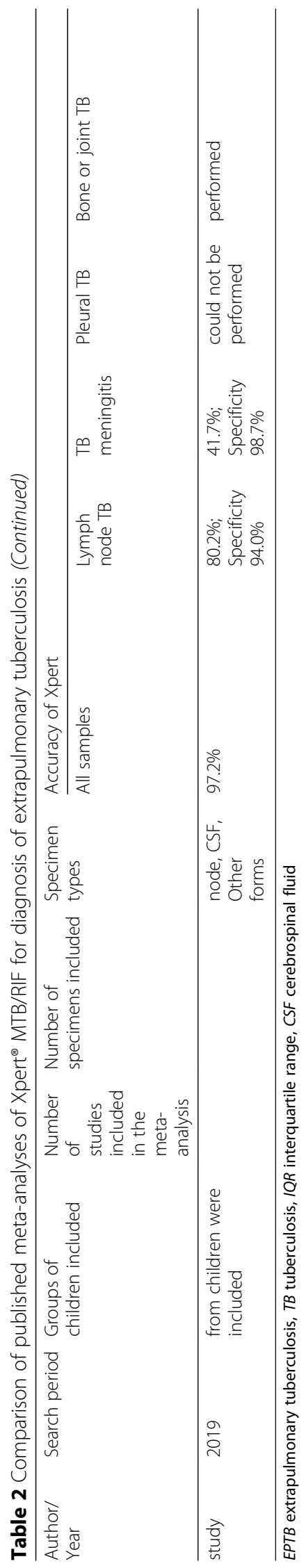


sensitivity was lower among children than among adults, particularly in CSF samples (42\% vs. $71 \%$ ) [10]. In the case of musculoskeletal $\mathrm{TB}$ and pleural $\mathrm{TB}$, the data were insufficient to carry out meta-analysis to determine the diagnostic accuracy of Xpert in pediatric populations, as two studies of musculoskeletal TB used different sample types (tissue vs. fluid), and only one study used pleural TB samples [20, 24]. However, these studies also displayed lower sensitivity in children than in adults, likely because the sample volume that can be collected from children is relatively lower than that from adults and because of the paucibacillary nature of EPTB in the former [21, 23, 26]. For CSF samples, a high sample volume was shown to increase the sensitivity of Xpert [27]. The total number of TB bacilli in the test sample plays an important role in the sensitivity of Xpert [27, 28], indicating that the sensitivity of Xpert in liquid samples may be lower than expected.

Since 2013, the WHO has recommended Xpert rather than conventional tests for the diagnosis of TB meningitis and TB lymphadenitis in children. As per the 2013 WHO data, the pooled sensitivity and specificity of lymph node TB in children were $86 \%$ (95\% CI $0.65-$ 0.96 ) and $81 \%$ (95\% CI $0.54--0.93)$, respectively. In the case of TB meningitis, the pooled specificity was $95 \%$ (95\% CI 0.81-0.99) and sensitivity could not be determined because of insufficient data [8]. To our knowledge, this is the first meta-analysis to evaluate the sensitivity of Xpert for TB meningitis only in pediatric populations. Our results suggest that negative Xpert results in children should be interpreted with caution with respect to ruling out pediatric TB meningitis. However, because TB meningitis is potentially lethal in children, the rapidity of Xpert explains why it should be used as an initial diagnostic test for TB meningitis despite its low sensitivity.

Recently, the next-generation Xpert MTB/RIF assay, Xpert MTB/RIF Ultra assay (Cepheid) (ULTRA), was developed; its limit of detection was enhanced by $\sim 8$-fold compared to the previous Xpert MTB/RIF assay and it includes a larger chamber and additional molecular targets [29]. In 2017, the WHO recommended the use of ULTRA as a replacement for Xpert MTB/RIF in all settings [30]. A single study of TB meningitis conducted in Uganda assessed the diagnostic accuracy of Xpert and ULTRA compared to CRS based on a positive CSF culture, Xpert, or ULTRA results. The sensitivity of detection of MTB for CSF was 95\% for ULTRA (21 of 22) relative to $45 \%$ for Xpert MTB/RIF (10 of 22) [28]. ULTRA is expected to have higher sensitivity for EPTB. However, as discussed herein, pediatric EPTB samples showed lower sensitivity than adult samples. ULTRA would be helpful for the paucibacillary population, particularly in terms of diagnostic sensitivity. However, considering that the sensitivity of the previous version of Xpert is low in children and yielded different values among samples, further studies are required to determine the reliability of the negative results.

EPTB is common in children but difficult to diagnose because the sampling methods are invasive. A point-ofcare ultrasound (POCUS) protocol (focused assessment with sonography for HIV-associated TB, FASH) is a noninvasive diagnostic tool developed to improve the diagnosis of EPTB in HIV-infected adults [31]. Although few studies have been conducted in children, POCUS has been evaluated for the diagnosis of pediatric EPTB, with some studies showing meaningful results in children [32]. A combination of various diagnostic methods such as Xpert and POCUS may improve the accuracy of diagnosing pediatric EPTB.

The present meta-analysis revealed high heterogeneity in patients with TB lymphadenitis and meningitis. Although the population was limited to those of the pediatric age and the samples were divided into subgroups, differences in the processing methods of samples and the small sample size may have resulted in high heterogeneity.

\section{Conclusion}

In diagnosing pediatric EPTB, Xpert displayed high specificity regardless of the specimen type, but exhibited modest sensitivity, which varied among specimen types. Particularly, in CSF samples, Xpert displayed the lowest sensitivity compared to the CRS. Although positive Xpert results can be considered to indicate presumptive EPTB in children, EPTB cannot be ruled out based on negative test results. Future clinical trials are required to expand the evidence for using Xpert to diagnose pediatric EPTB with different forms of extrapulmonary specimens in various clinical settings.

\section{Supplementary information}

Supplementary information accompanies this paper at https://doi.org/10. 1186/s12879-019-4745-1.

Additional file 1. Study search strategy

\section{Abbreviations}

Cl: Confidence interval; CRS: Composite reference standard; CSF: Cerebrospinal fluid; EPTB: Extrapulmonary tuberculosis; FNAB: Fine needle aspirationbiopsy; LN: Lymph node; ROC: Receiver operating characteristic; TB: Tuberculosis; ULTRA: Xpert MTB/RIF Ultra assay

\section{Acknowledgements \\ Not applicable.}

\section{Authors' contributions}

JGA devised the review, main conceptual ideas, and proof outline. YSS primarily collected the data, performed preliminary data analysis, and wrote the manuscript. JMK and DSK participated in the study design and data evaluation. JGA supervised the design and execution of the study, 
interpreted the final data, and contributed to the writing of the manuscript All the authors have approved the manuscript for publication.

\section{Funding}

The present study was supported by a 2017 grant from the Korean Society of Pediatric Infectious Diseases. The funder did not participate in study design, data collection, data analysis, decision to publish, or manuscript preparation.

\section{Availability of data and materials}

The data used in the present study are appropriately cited.

\section{Ethics approval and consent to participate}

No institutional review board approval or ethics statement was required as the present meta-analysis followed a retrospective design.

\section{Consent for publication}

Not applicable.

\section{Competing interests}

The authors declare no conflicts of interests.

Received: 3 September 2019 Accepted: 28 December 2019

Published online: 06 January 2020

\section{References}

1. Global tuberculosis report 2019. In Geneva: World Health Organization; 2019.

2. Lee JY. Diagnosis and treatment of extrapulmonary tuberculosis. Tuberc Respir Dis. 2015;78(2):47-55.

3. Peto HM, Pratt RH, Harrington TA, LoBue PA, Armstrong LR. Epidemiology of extrapulmonary tuberculosis in the United States, 1993-2006. Clin Infect Dis. 2009;49(9):1350-7.

4. Nelson LJ, Wells CD. Global epidemiology of childhood tuberculosis. Int J Tuberc Lung Dis. 2004;8(5):636-47.

5. Denkinger CM, Schumacher SG, Boehme CC, Dendukuri N, Pai M, Steingart KR. Xpert MTB/RIF assay for the diagnosis of extrapulmonary tuberculosis: a systematic review and meta-analysis. Eur Respir J. 2014;44(2):435-46.

6. Purohit M, Mustafa T. Laboratory diagnosis of extra-pulmonary tuberculosis (EPTB) in resource-constrained setting: state of the art, challenges and the need. J Clin Diagn Res: JCDR. 2015;9(4):EE01-E6.

7. Wu XR, Yin QQ, Jiao AX, Xu BP, Sun L, Jiao WW, Xiao J, Miao Q, Shen C, Liu F, et al. Pediatric tuberculosis at Beijing Children's hospital: 2002-2010. Pediatrics. 2012;130(6):e1433-40.

8. Automated Real-Time Nucleic Acid Amplification Technology for Rapid and Simultaneous Detection of Tuberculosis and Rifampicin Resistance: Xpert MTB/RIF Assay for the Diagnosis of Pulmonary and Extrapulmonary TB in Adults and Children: Policy Update. In. WHO Guidelines Approved by the Guidelines Review Committee. Geneva: World Health Organization; 2013.

9. Chang K, Lu W, Wang J, Zhang K, Jia S, Li F, Deng S, Chen M. Rapid and effective diagnosis of tuberculosis and rifampicin resistance with Xpert MTB/ RIF assay: a meta-analysis. J Inf Secur. 2012;64(6):580-8.

10. Kohli M, Schiller I, Dendukuri N, Dheda K, Denkinger CM, Schumacher SG, Steingart KR. Xpert((R)) MTB/RIF assay for extrapulmonary tuberculosis and rifampicin resistance. Cochrane Database Syst Rev. 2018;8:Cd012768.

11. Li S, Liu B, Peng M, Chen M, Yin W, Tang H, Luo Y, Hu P, Ren H. Diagnostic accuracy of Xpert MTB/RIF for tuberculosis detection in different regions with different endemic burden: A systematic review and meta-analysis. PloS One. 2017;12(7):e0180725-e.

12. Maynard-Smith L, Larke N, Peters JA, Lawn SD. Diagnostic accuracy of the Xpert MTB/RIF assay for extrapulmonary and pulmonary tuberculosis when testing non-respiratory samples: a systematic review. BMC Infect Dis. 2014; 14:709.

13. Penz E, Boffa J, Roberts DJ, Fisher D, Cooper R, Ronksley PE, James MT. Diagnostic accuracy of the Xpert(R) MTB/RIF assay for extra-pulmonary tuberculosis: a meta-analysis. Int J Tuberc Lung Dis. 2015;19(3):278-84 i-iii.

14. Sehgal IS, Dhooria S, Aggarwal AN, Behera D, Agarwal R. Diagnostic performance of Xpert MTB/RIF in Tuberculous pleural effusion: systematic review and meta-analysis. J Clin Microbiol. 2016;54(4):1133-6.
15. Shen $Y, Y u$ G, Zhong F, Kong X. Diagnostic accuracy of the Xpert MTB/RIF assay for bone and joint tuberculosis: a meta-analysis. PLoS One. 2019;14(8): e0221427.

16. Yu G, Zhong F, Ye B, Xu X, Chen D, Shen Y. Diagnostic accuracy of the Xpert MTB/RIF assay for lymph node tuberculosis: a systematic review and meta-analysis. Biomed Res Int. 2019;2019:4878240.

17. Whiting PF, Rutjes AW, Westwood ME, Mallett S, Deeks JJ, Reitsma JB, Leeflang MM, Sterne JA, Bossuyt PM. QUADAS-2: a revised tool for the quality assessment of diagnostic accuracy studies. Ann Intern Med. 2011; 155(8):529-36.

18. Bholla M, Kapalata N, Masika E, Chande H, Jugheli L, Sasamalo M, Glass TR, Beck HP, Reither K. Evaluation of Xpert(R) MTB/RIF and Ustar EasyNAT TB IAD for diagnosis of tuberculous lymphadenitis of children in Tanzania: a prospective descriptive study. BMC Infect Dis. 2016;16:246.

19. Coetzee L, Nicol MP, Jacobson R, Schubert PT, van Helden PD, Warren RM, Wright CA. Rapid diagnosis of pediatric mycobacterial lymphadenitis using fine needle aspiration biopsy. Pediatr Infect Dis J. 2014;33(9):893-6.

20. Held M, Laubscher M, Mears S, Dix-Peek S, Workman L, Zar H, Dunn R. Diagnostic accuracy of the Xpert MTB/RIF assay for Extrapulmonary tuberculosis in children with musculoskeletal infections. Pediatr Infect Dis J. 2016;35(11):1165-8.

21. Solomons RS, Visser DH, Friedrich SO, Diacon AH, Hoek KG, Marais BJ, Schoeman JF, van Furth AM. Improved diagnosis of childhood tuberculous meningitis using more than one nucleic acid amplification test. Int J Tuberc Lung Dis. 2015;19(1):74-80.

22. Vadwai V, Boehme C, Nabeta P, Shetty A, Alland D, Rodrigues C. Xpert MTB/ RIF: a new pillar in diagnosis of extrapulmonary tuberculosis? J Clin Microbiol. 2011;49(7):2540-5.

23. Das A, Anupurba S, Mishra OP, Banerjee T, Tripathi R. Evaluation of Xpert MTB/RIF assay for diagnosis of tuberculosis in children. J Trop Pediatr. 2019; 65(1):14-20.

24. Kim YW, Kwak N, Seong MW, Kim EC, Yoo CG, Kim YW, Han SK, Yim JJ. Accuracy of the Xpert(R) MTB/RIF assay for the diagnosis of extra-pulmonary tuberculosis in South Korea. Int J Tuberc Lung Dis. 2015;19(1):81-6.

25. Tortoli E, Russo C, Piersimoni C, Mazzola E, Dal Monte P, Pascarella M, Borroni E, Mondo A, Piana F, Scarparo C, et al. Clinical validation of Xpert MTB/RIF for the diagnosis of extrapulmonary tuberculosis. Eur Respir J. 2012; 40(2):442-7

26. Detjen AK, DiNardo AR, Leyden J, Steingart KR, Menzies D, Schiller I, Dendukuri N, Mandalakas AM. Xpert MTB/RIF assay for the diagnosis of pulmonary tuberculosis in children: a systematic review and meta-analysis. Lancet Respir Med. 2015:3(6):451-61.

27. Nhu NT, Heemskerk D. Thu do DA, Chau TT, Mai NT, Nghia HD, Loc PP, ha DT, Merson L, Thinh TT, et al. evaluation of GeneXpert MTB/RIF for diagnosis of tuberculous meningitis. J Clin Microbiol. 2014;52(1):226-33.

28. Bahr NC, Nuwagira E, Evans EE, Cresswell FV, Bystrom PV, Byamukama A, Bridge SC, Bangdiwala AS, Meya DB, Denkinger CM, et al. Diagnostic accuracy of Xpert MTB/RIF Ultra for tuberculous meningitis in HIV-infected adults: a prospective cohort study. Lancet Infect Dis. 2018;18(1):68-75.

29. Chakravorty S, Simmons AM, Rowneki M, Parmar H, Cao Y, Ryan J, Banada PP, Deshpande S, Shenai S, Gall A, et al. The New Xpert MTB/RIF Ultra: Improving Detection of Mycobacterium tuberculosis and Resistance to Rifampin in an Assay Suitable for Point-of-Care Testing. MBio. 2017;8(4): e00812-00817.

30. Non-inferiority analysis of Xpert MTF/RIF Ultra compared to Xpert MTB/RIF. In. WHO meeting report of a technical expert consultation. Geneva: World Health Organization; 2017.

31. Heller T, Wallrauch C, Goblirsch S, Brunetti E. Focused assessment with sonography for HIV-associated tuberculosis (FASH): a short protocol and a pictorial review. Crit Ultrasound J. 2012;4(1):21.

32. Belard S, Heuvelings CC, Banderker E, Bateman L, Heller T, Andronikou S, Workman L, Grobusch MP, Zar HJ. Utility of point-of-care ultrasound in children with pulmonary tuberculosis. Pediatr Infect Dis J. 2018;37(7):637-42.

\section{Publisher's Note}

Springer Nature remains neutral with regard to jurisdictional claims in published maps and institutional affiliations. 